\title{
NUMERICAL FINITE ELEMENT METHOD HOMOGENIZATION OF COMPOSITE MATERIALS REINFORCED WITH FIBERS
}

The paper presents the micromechanical modelling of fiber-reinforced composites in order to determine elastic properties of the homogenized material. For this purpose implementation of homogenization theory was required and analyses were performed. The polymer matrix of three-dimensional representative volume element (RVE) of the composites is modelled by the finite element method (FEM). Software for homogenization of material properties uses direct homogenization method which is based on volume average of stresses in the RVE. Homogenization of composite plate is performed by linking MATLAB and ANSYS software. Calculated elastic properties of the homogenized material are given for epoxy matrix reinforced with carbon, fiberglass and kevlar fiber material.

Keywords: Fiber-reinforced composites, Representative volume element, Finite element method.

\section{Introduction}

Modern materials are characterized by a wide spectrum of tailored mechanical, optical, magnetic, electronic, or thermomechanical properties. Using of laminated composites allows the designer to optimize material/structural systems which complicates their analysis. The prediction of the macroscopic stress-strain response of the composite material is related to the description of its complex microstructural behavior [1 and 2] Some analytical and numerical techniques have been used for prediction and characterization of composite microstructure behavior [3]. Analytical methods provide reasonable prediction for relatively simple configurations of the phases. Complicated geometries, loading conditions and material properties often do not yield analytical solutions, due to complexity and number of equations. In this case, various numerical methods [4 - 7] are used for approximate solving, but they still make some simplifying assumptions about the microstructures of heterogeneous multiphase materials.

In homogenization process the original heterogeneous material is replaced by homogeneous material with the same mechanical properties. In other words, homogenization and averaging of properties and micro-fields of the material are considered as a homogeneous equivalent medium at the macrolevel, and the effective properties of the medium are determined on the basis of the analysis of the microstructure, micro-geometry and properties of the materials.
There are various homogenization methods. Direct homogenization is based on the volume average of field variables, such as stress, strain and energy density. Effective properties can be calculated from effective properties definitions. The average and calculation of field variables can be performed numerically, for example, by finite element method (FEM) or boundary element method and geometry and microstructural properties can be generalized for real composite materials which do not have periodic structure distribution of the fibers in the matrix [8].

Indirect homogenization is based on the Eshelby solution of self-deformation for one inclusion in an infinite matrix - the equivalent inclusion method [9]. An alternative approach to direct and indirect homogenization is the variational method which can determine the upper and lower limits of the elasticity modulus [10].

A relatively new approach for homogenization of microstructures consists of mathematical homogenization based on a two-scale extension of the displacement field [11].

\section{Methodology}

The composite under consideration is constituted by continuous and parallel cylindrical fibers with equal radius and centers periodically spaced in a hexagonal and a squared array, as it appears in Fig. 1. We assumed an ideal cohesion between the fiber and the matrix. A procedure of homogenization

\footnotetext{
* Daniel Riecky, Milan Zmindak, Zoran Pelagic

Department of Applied Mechanics, Faculty of Mechanical Engineering, University of Zilina, Slovakia

E-mail: milan.zmindak@fstroj.uniza.sk
} 
of material properties of composites using the method of representative volume element RVE was used. For the analysis of material properties an own software in MATLAB language was programmed and a part of the solution was carried out in ANSYS software. The RVE consists of volume elements SOLID45 (Fig. 2 ) and then it is loaded by unit strains in various directions. The effective lamina properties are obtained from the volume means of stress values obtained by loading of the RVE.

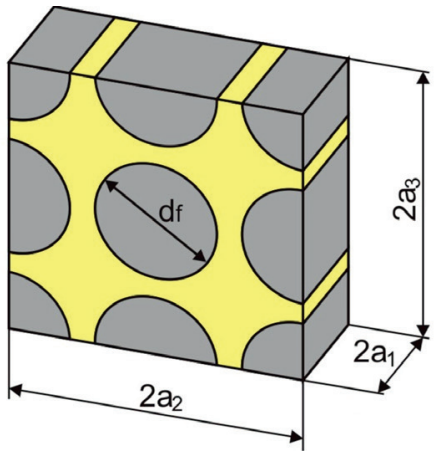

a)

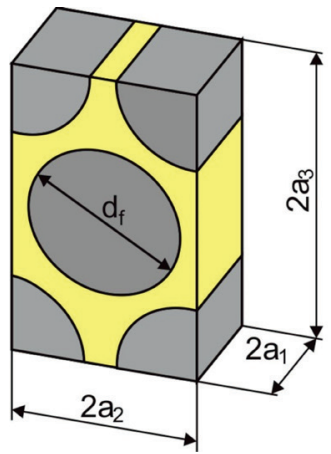

b)
Fig. 1 Representative volume elements, a) square configuration, b) hexagonal configuration

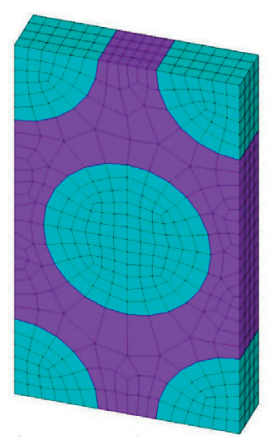

a)

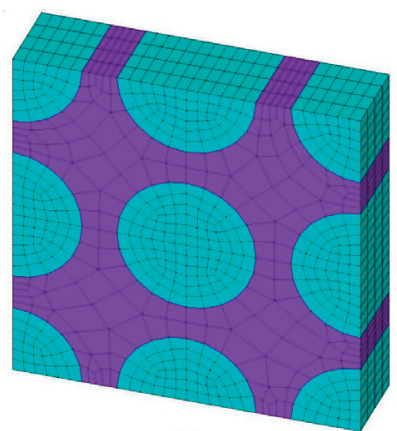

b)
Fig. 2 The finite element mesh adopted in the computation a) square configuration, b) hexagonal configuration

Homogenized lamina RVE consists of fibers and epoxy matrix. The fibers are from three material types: carbon, glass, polyaramide. Used carbon fibers have an industrial label T300 and M40J. The glass fiber label is EGlass and S2Glass. Polyaramide fibers have the label K49. Fiber material properties are listed in Table 1 and the matrix properties are listed in Table 2.
Fiber material properties

Table 1

\begin{tabular}{|c|c|c|c|}
\hline \multirow{2}{*}{} & \multicolumn{3}{|c|}{ Fiber material } \\
\cline { 2 - 4 } & Carbon M40J & $\begin{array}{c}\text { Fiberglass } \\
\text { S2Glass }\end{array}$ & Kevlar K49 \\
\hline$E_{f}[\mathrm{GPa}]$ & 377 & 85.5 & 135.5 \\
\hline$F_{l t}[\mathrm{GPa}]$ & 4.41 & 4.6 & 3.53 \\
\hline$v$ & 0.33 & 0.22 & 0.37 \\
\hline$\rho_{m}\left[\mathrm{~kg} / \mathrm{m}^{3}\right]$ & 1770 & 2490 & 1450 \\
\hline$d_{f}[\mu \mathrm{m}]$ & 5 & 10 & 10 \\
\hline
\end{tabular}

Matrix material properties

Table 2

\begin{tabular}{|c|c|c|c|c|c|}
\hline $\begin{array}{c}\text { Matrix } \\
\text { material }\end{array}$ & $E_{m}[\mathrm{GPa}]$ & $F_{l t}[\mathrm{MPa}]$ & $v$ & $\rho_{f}\left[\mathrm{~kg} / \mathrm{m}^{3}\right]$ & $G_{m}[\mathrm{GPa}]$ \\
\hline Epoxy & 3.45 & 70 & 0.3 & 85.5 & 1.33 \\
\hline
\end{tabular}

where subscript " $f$ " denotes fiber and " $m$ " matrix, respectively and

$E$ - Young modulus

$G$ - Shear modulus

$v$ - Poisson number

$F_{l t}$ - Longitudinal tensile strength

$\rho$ - Density

The RVE dimensions are calculated for the square fiber configuration - Fig. 1a, from the relations

$a_{2}=\sqrt{\frac{1}{4} \frac{\pi d_{f}^{2}}{V_{f}}}, a_{3}=a_{2}, a_{1}=0.5 a_{2}$

and for a hexagonal fiber configuration the RVE dimensions are in Fig. 1b, from the relations

$a_{2}=\sqrt{\frac{1}{8} \frac{\pi d^{2}}{V_{f} \operatorname{tg}\left(60^{\circ}\right)}}, a_{3}=a_{2} \operatorname{tg}\left(60^{\circ}\right)$,

$a_{1}=0.5 a_{2}$

where $a_{1}$ is the $x$-direction, in this case the fiber direction, $a_{2}$ is the $y$-direction, orthogonal to the fiber direction, $a_{3}$ is $z$-direction, transverse vertical to the fiber direction and $V_{f}$ is fiber volume fraction.

Analysis of microstructure directly yields a transversely isotropic stiffness tensor

$\left\{\begin{array}{c}\bar{\sigma}_{11} \\ \bar{\sigma}_{22} \\ \bar{\sigma}_{33} \\ \bar{\sigma}_{23} \\ \bar{\sigma}_{13} \\ \bar{\sigma}_{12}\end{array}\right\}=\left[\begin{array}{cccccc}C_{11} & C_{12} & C_{12} & 0 & 0 & 0 \\ C_{12} & C_{22} & C_{23} & 0 & 0 & 0 \\ C_{12} & C_{23} & C_{22} & 0 & 0 & 0 \\ 0 & 0 & 0 & 1 / 2\left(C_{22}-C_{23}\right) & 0 & 0 \\ 0 & 0 & 0 & 0 & C_{66} & 0 \\ 0 & 0 & 0 & 0 & 0 & C_{66}\end{array}\right]\left\{\begin{array}{c}\bar{\varepsilon}_{11} \\ \bar{\varepsilon}_{22} \\ \bar{\varepsilon}_{33} \\ \bar{\gamma}_{23} \\ \bar{\gamma}_{13} \\ \bar{\gamma}_{12}\end{array}\right\}$

where the 1-axis is aligned with the fiber direction and the overbar indicates the average computed over the volume RVE. The components of the tensor $C$ are determined by solving three elastic models of RVE with parameters $\left(a_{1}, a_{2}, a_{3}\right)$, subjected to the boundary conditions on the following displacement components. 


$$
\begin{aligned}
& u_{1}\left(a_{1}, y, z\right)-u_{1}\left(-a_{1}, y, z\right)=2 a_{1} \varepsilon_{i 1}^{0}, \\
& -a_{2} \leq y \leq a_{2} \\
& -a_{3} \leq z \leq a_{3} \\
& u_{1}\left(x, a_{2}, z\right)-u_{1}\left(x,-a_{2}, z\right)=2 a_{2} \varepsilon_{i 2}^{0}, \\
& -a_{1} \leq x \leq a_{1} \\
& -a_{3} \leq z \leq a_{3} \\
& u_{1}\left(x, y, a_{3}\right)-u_{1}\left(x, y,-a_{3}\right)=2 a_{3} \varepsilon_{i 3}^{0}, \\
& -a_{1} \leq x \leq a_{1} \\
& -a_{2} \leq y \leq a_{2}
\end{aligned}
$$

For the homogeneous composite material, the relationship between average stress and strain is

$$
\bar{\sigma}_{\alpha}=C_{\alpha \beta} \overline{\mathcal{E}}_{\beta}
$$

$\alpha, \beta=1, \ldots, 6$ is the contracted notation given in [12]. This implies that elements of the matrix $C$ are determined by solution of six elastic RVE models in which boundary conditions (4-6) are applied for only one component of the strain $\varepsilon_{\beta}^{0}$. This component is different from zero for each of the six problems. Once the components of the transversely isotropic tensor $C$ are known, the five elastic properties of the homogenized material can be computed by [13]:

$$
\begin{aligned}
& E_{1}=C_{11}-2 C_{12}^{2} /\left(C_{22}+C_{23}\right) \\
& v_{12}=C_{12} /\left(C_{22}+C_{23}\right) \\
& E_{2}=\left(C_{11}\left(C_{22}+C_{23}\right)-2 C_{12}^{2}\right)\left(C_{22}-C_{23}\right) /\left(C_{11} C_{22}-C_{12}^{2}\right) \\
& v_{23}=\left(C_{11} C_{23}-C_{12}^{2}\right) /\left(C_{11} C_{22}-C_{12}^{2}\right) \\
& G_{12}=C_{66}
\end{aligned}
$$

where $E_{1}$ and $E_{2}$ are longitudinal and transversal Young's moduli, $v_{12}$ and $v_{23}$ are longitudinal and transversal Poisson's ratios and $G_{12}$ is the longitudinal shear modulus. The shear modulus $G_{23}$ in the transversal plane can be obtained using classical relation between Young modulus $E$ and shear modulus $G$, so

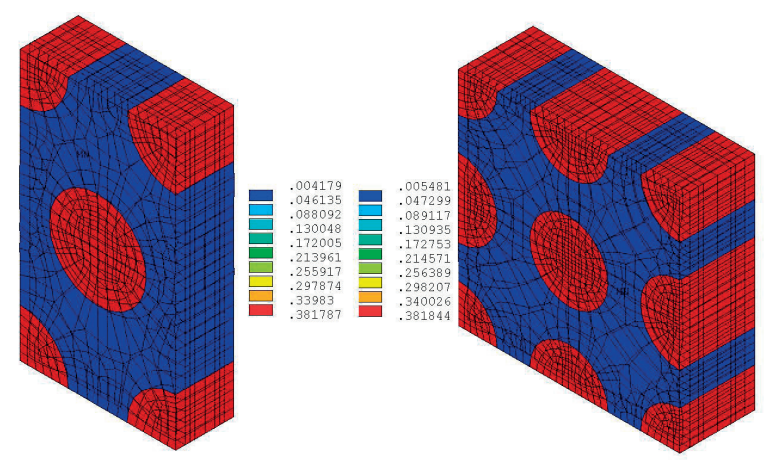

$G_{23}=C_{44}=\frac{1}{2}\left(C_{22}-C_{23}\right)=\frac{E_{2}}{2\left(1+v_{23}\right)}$

In order to evaluate the elastic matrix $C$ of the composite, the RVE is subjected to an average strain $\bar{\varepsilon}_{\beta}$. The unit strain applied on the boundary results in a complex state of stress in the RVE.

Then the volume average of the strain in the RVE equals to the applied strain

$\overline{\mathcal{E}}_{i j}=\frac{1}{V} \int_{V} \varepsilon_{i j} d V=\varepsilon_{i j}^{0}$

Then volume average of stress in RVE equals to required components of the elastic matrix as

$C_{i j}=\bar{\sigma}_{i}=\frac{1}{V} \int_{V} \sigma_{i} d V$

The coefficients in $C$ are found by setting a different problem for each column of $C$ and the components. Details of the procedure for calculation of the coefficients of the matrix $C$ are given in [14].

\section{Results}

Homogenization of a composite plate is performed by linking MATLAB and ANSYS software. Homogenization of the material properties were done for fiber volume fractions $V_{f}$ from the interval $\langle 0.2,0.6\rangle$. The entire process is automated requires just entering a type of fibers, their arrangement and the step increment volume fraction. The finite element code ANSYS 11.0 is used to solve the problem described above. The matrix and fibers are modeled by linear elastic isoparametric brick elements with eight nodes and six faces (i.e. the ANSYS SOLID elements). FE mesh is symmetric with respect to the coordinate planes.

In Figs. 3 to 6 deformed shapes and contour plots of stresses for different strains applied to stretch the RVEs are described. The boundary conditions in the calculation of the sixth column of $C$ are enforced by using coupling constraint equations (CE).

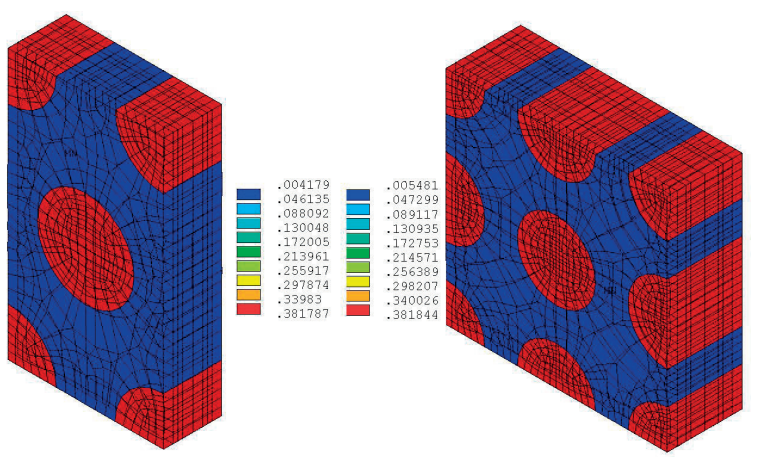

Fig. 3 Deformed shape and contour plot of stress $\sigma_{11}, \varepsilon_{1}^{0}=1, \mathcal{E}_{2}^{0}=\varepsilon_{3}^{0}=\gamma_{4}^{0}=\gamma_{5}^{0}=\gamma_{6}^{0}=0$ 

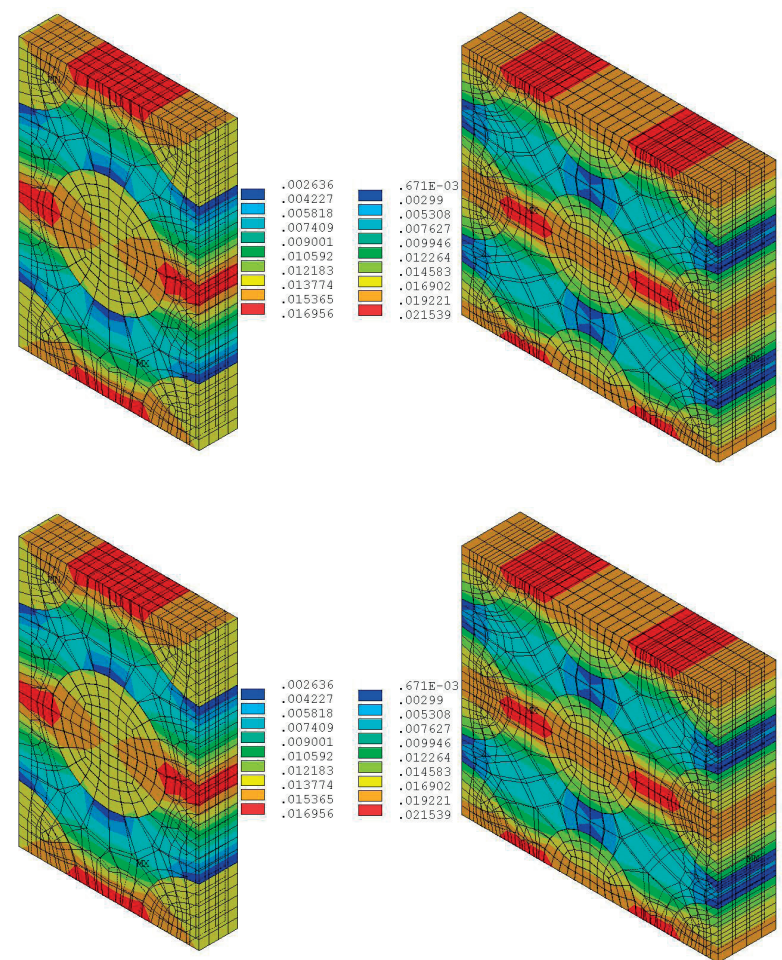

Fig. 4 Deformed shape and contour plot of stress $\sigma_{22}$, $\varepsilon_{2}^{0}=1, \varepsilon_{1}^{0}=\varepsilon_{3}^{0}=\gamma_{4}^{0}=\gamma_{5}^{0}=\gamma_{6}^{0}=0$
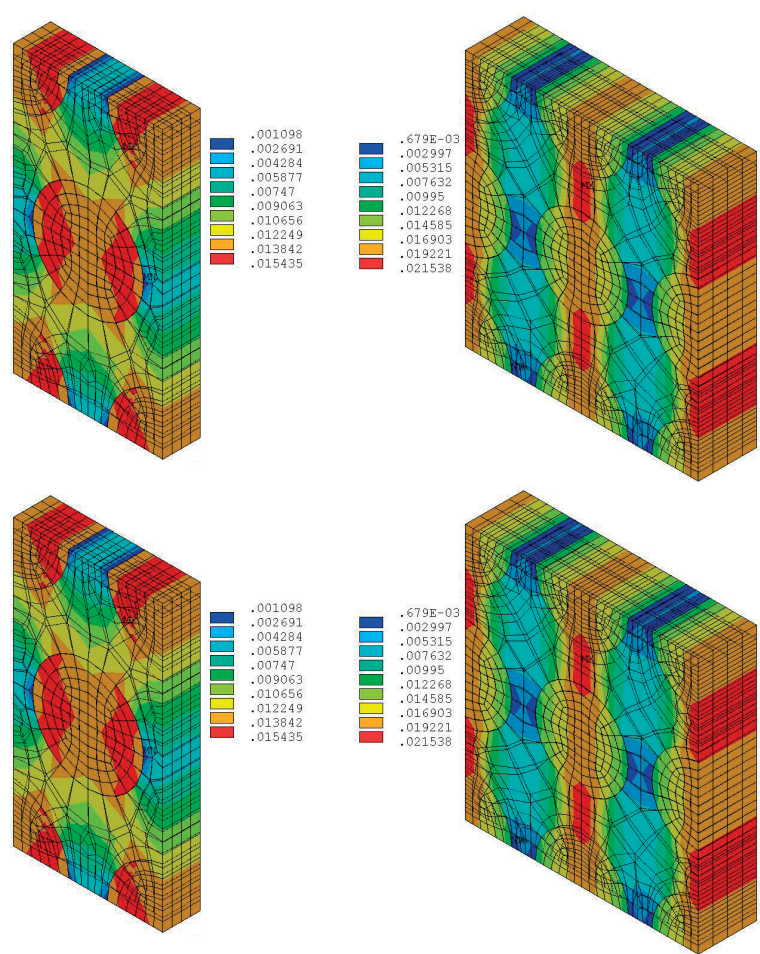

Fig. 5 Deformed shape and contour plot of stress $\sigma_{33}$, $\varepsilon_{3}^{0}=1, \varepsilon_{1}^{0}=\varepsilon_{2}^{0}=\gamma_{4}^{0}=\gamma_{5}^{0}=\gamma_{6}^{0}=0$
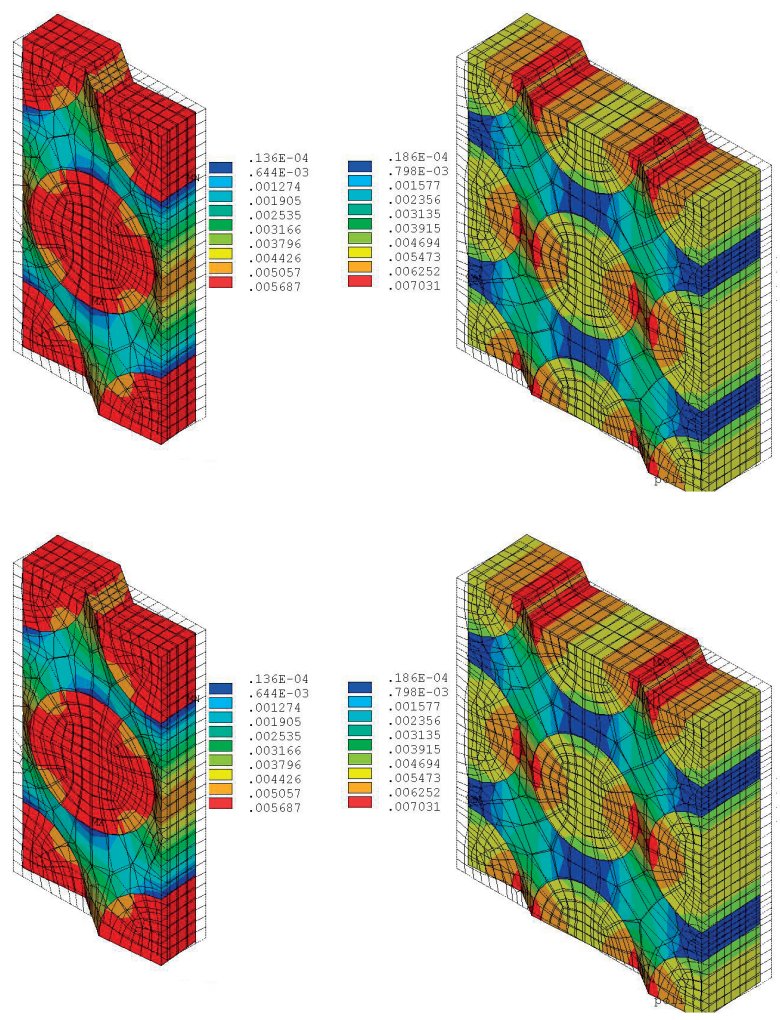

Fig. 6 Deformed shape and contour plot of stress $\tau_{13}$, $\gamma_{6}^{0}=\varepsilon_{12}^{0}+\varepsilon_{21}^{0}=1, \varepsilon_{1}^{0}=\varepsilon_{2}^{0}=\varepsilon_{3}^{0}=\gamma_{4}^{0}=\gamma_{5}^{0}=0$
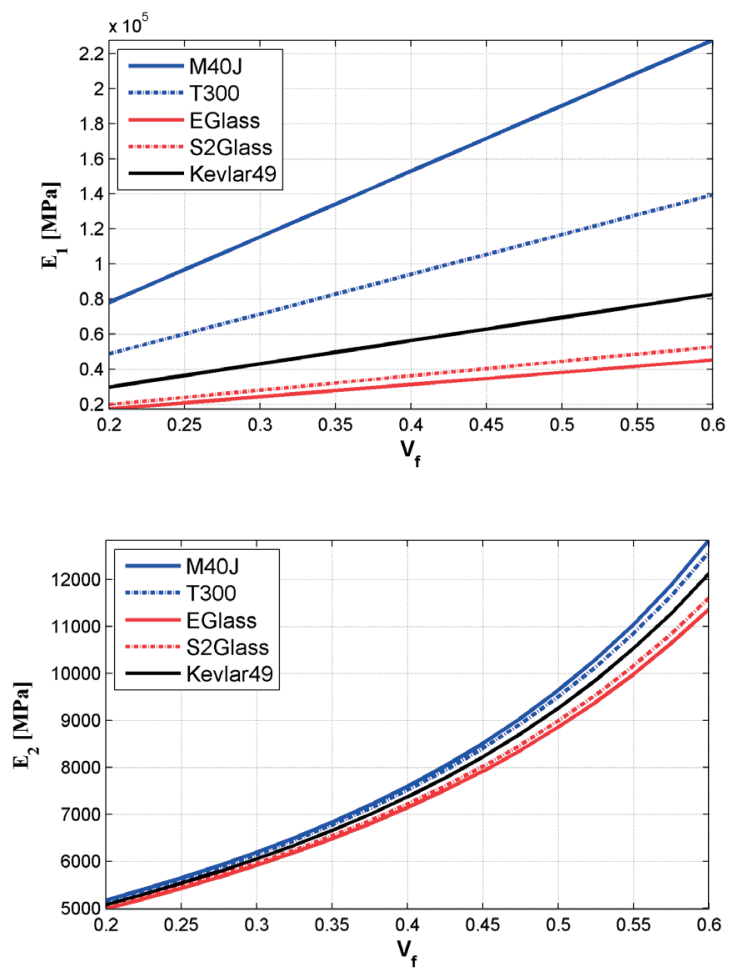

Fig. 7 Effective elastic constants $E_{1}$ and $E_{2}$ vs. $V_{f}$ 

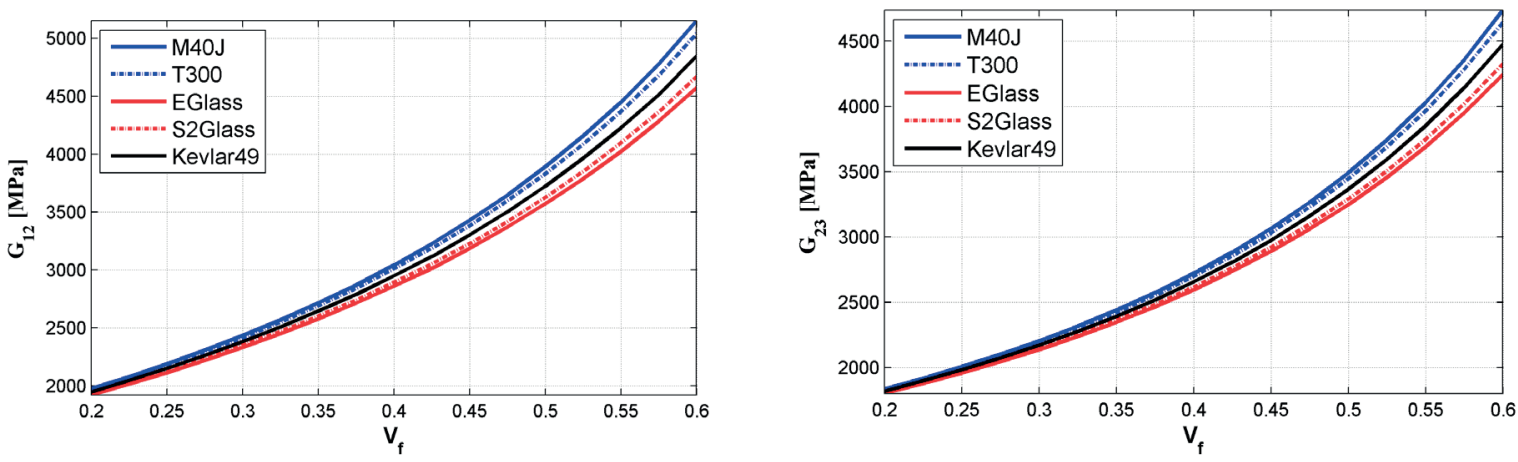

Fig. 8 Effective elastic constants $G_{12}$ and $G_{23}$ vs. $V_{f}$
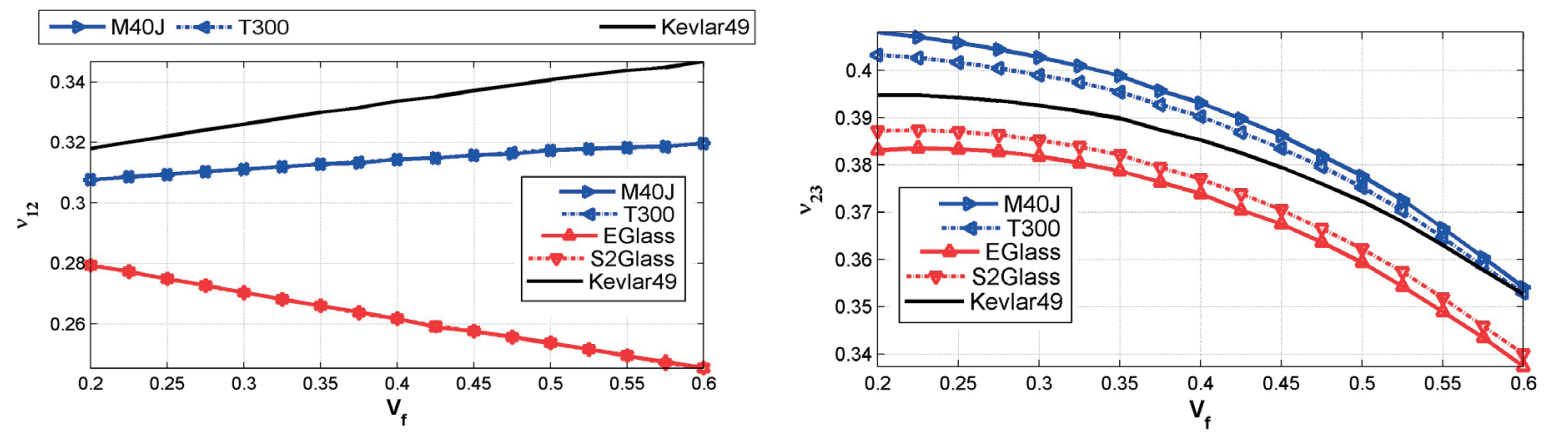

Fig. 9 Effective elastic constants $v_{12}$ and $v_{23}$ vs. $V_{f}$

Calculated elastic properties of the composite with fiber volume fraction $V_{f}=0.6$

Table 3

\begin{tabular}{|c|c|c|c|c|c|c|}
\hline \multirow{2}{*}{$\mathrm{V}_{\mathrm{f}}=0.6$} & \multicolumn{2}{|c|}{ M40J } & \multicolumn{2}{c|}{ S2Glass } & \multicolumn{2}{c|}{ K49 } \\
\cline { 2 - 7 } & $\mathrm{h}$ & $\mathrm{s}$ & $\mathrm{h}$ & $\mathrm{s}$ & $\mathrm{h}$ & $\mathrm{s}$ \\
\hline $\mathrm{E}_{1}[\mathrm{GPa}]$ & 227.58 & 227.58 & 52.683 & 52.687 & 82.683 & 82.685 \\
\hline $\mathrm{E}_{2}[\mathrm{GPa}]$ & 12.831 & 16.71 & 11.607 & 14.334 & 12.121 & 15.301 \\
\hline $\mathrm{G}_{12}[\mathrm{GPa}]$ & 5.15 & 5.53 & 4.67 & 4.94 & 4.844 & 5.155 \\
\hline $\mathrm{G}_{23}[\mathrm{GPa}]$ & 4.737 & 6.967 & 4.3314 & 5.905 & 4.481 & 6.288 \\
\hline$v_{12}$ & 0.320 & 0.321 & 0.246 & 0.245 & 0.347 & 0.348 \\
\hline$v_{23}$ & 0.354 & 0.199 & 0.340 & 0.214 & 0.352 & 0.217 \\
\hline
\end{tabular}

In Figs. 7 to 9 courses of the homogenized elastic material properties for hexagonal array computed using equations (8) are described. Calculated elastic properties of the homogenized material for $\mathrm{V}_{\mathrm{f}}=0.6$ are given in Table 3 where the indices " $\mathrm{h}$ " and "s" denote hexagonal array and square array, respectively.

\section{Conclusions}

Accurate and efficient methods have been proposed within the framework of the displacement-based FEM for solving the unit cell homogenization (RVE) problem for periodic composites.
The modelling procedure is explained briefly in this paper. If one wishes to perform a parametric study, repeating this process on an interactive session, using the CAE graphical user interface (GUI) is very time consuming and prone to errors. Instead, it is possible to capture the ANSYS script generated by CAE during an interactive session and use it to automate the process.

\section{Acknowledgement}

The authors gratefully acknowledge support from the Slovak and Technology Assistance Agency registered under number APVV-0736-12, Slovak Grant Agency VEGA 1/1226/12. 


\section{References}

[1] CAPORAlE, A., LUCIANO, R., SACCO, E.: Micromechanical Analysis of Interfacial Debonding in Unidirectional Fiberreinforced Composites, Computers and Structures 84, 2006, 2200-2211.

[2] DEKYS, V., KOPAS, P., SAPIETA, M., STEVKA, O., A Detection of Deformation Mechanisms using Infrared Thermography and Acoustic Emission, Applied Mechanics and Materials, vol. 474, 2014, 315-320.

[3] ZMINDAK, M., NOVAK, P.: Special Approach for Thermal Modelling Fibre-reinfoced Composites with Larger Aspect Ratio, Communications - Scientific Letters of the University of Zilina, 4A/2012, 73-78.

[4] SOARES, D. JR., SLADEK, J., SLADEK, V., ZMINDAK, M., MEDVECKY, S: Porous Media Analysis by Modified MLPG Formulations, CMC: Computers, Materials \& Continua, vol. 27(2), 2012, 101-127.

[5] LACK T., GERLICI J.: Wheel/rail Contact Stress Evaluation by Means of the Modified Strip Method, Communications - Scientific Letters of the University of Zilina, No. 3, 2013, 126-132.

[6] BEDNAR, R., SAGA, M., VASKO, M.: Effectivity Analysis of Chosen Numerical Methods for Solution of Mechanical Systems with Uncertain Parameters. Communications - Scientific Letters of the University of Zilina, No. 4, 2013, 40-45.

[7] V. KOMPIS, Z. MURCINKOVA: Thermal Properties of Short Fiber Composites Modeled by Meshless Method, Advances in Materials Science and Engineering, vol. 2014, Article ID 521030, 8 p., http://dx.doi.org/10.1155/2014/521030

[8] POSPISIL, T.: Generating Non-periodic Microstructures of Fiber Composites. Engineering Mechanics, vol. 17, No. 5/6, 2010, 393405.

[9] ESHELBY, J. D.: The Determination of Elastic Field of an Ellipsoidal Inclusion and Related Problems, Proc. R. Soc. London, 1957, 276-396.

[10] HASHIN, Z., SHTRIKMAN, S.: On Some Variational Principles in Anisotropic and Nonhomogeneous Elasticity, J. Mech. Phys. Solids, vol. 10, 1962, 335-342.

[11] KOUZNETSOVA, V. G.: Computational Homogenization for the Multi-scale Analysis of Multi-phase materials, TU Eindhoven, 2012.

[12] BARBERO, E. J.: Finite Element Analysis of Composite Materials, CRC Press : Boca Raton, 2007.

[13] REDDY, J. N.: Mechanics of Laminated Composite Plates and Shells: Theory and Analysis, $2^{\text {nd }}$ ed., CRC Press, 2004.

[14] KORMANIKOVA, E., RIECKY, D., ZMINDAK, M.: Strength of Composites with Short Fibers. In: Eds, J. Murin: Computational Modelling and Advanced Simulations, Series: Computational Methods in Applied Sciences, vol. 24, 2011. 